\title{
Comorbid personality disorders among patients with depression
}

\author{
This article was published in the following Dove Press journal: \\ Neuropsychiatric Disease and Treatment \\ 17 April 2015 \\ Number of times this article has been viewed
}

\author{
Nahathai Wongpakaran \\ Tinakon Wongpakaran \\ Vudhichai Boonyanaruthee \\ Manee Pinyopornpanish \\ Suthi Intaprasert \\ Department of Psychiatry, Faculty \\ of Medicine, Chiang Mai University, \\ Chiang Mai, Thailand
}

Purpose: To investigate the personality disorders (PDs) diagnosed in patients with depressive disorders.

Material and methods: This study included a cross-sectional analysis, and was an extension of the Thai Study of Affective Disorder (THAISAD) project. Eighty-five outpatients with depressive disorders were interviewed using the Mini International Neuropsychiatric Inventory to assess for depression, in accordance with the Diagnostic and Statistical Manual of Mental Disorders, Fourth Edition, Text Revision and using the Thai version of the Structured Clinical Interview for PDs to assess for PD.

Results: Seventy-seven percent of the patients had at least one PD, 40\% had one PD and $60 \%$ had two or more PDs (mixed cluster). The most common PDs found were borderline PD (20\%) and obsessive-compulsive PD (10.6\%), while the occurrence of avoidant PD was low when compared to the findings of previous, related studies. Among the mixed cluster, cluster A combined with cluster $\mathrm{C}$ was the common mix. Both dysthymic disorder and double depression were found to have a higher proportion of PDs than major depressive disorder $(85.7 \%$ versus 76.1\%). Dependent PD was found to be less common in this study than in previous studies, including those carried out in Asia.

Conclusion: The prevalence of PDs among those with depressive disorder varied, and only borderline PD seems to be consistently high within and across cultures. Mixed cluster plays a prominent role in depression, so more attention should be paid to patients in this category.

Keywords: personality disorders, depressive disorder, prevalence, Asian, mixed cluster, SCID-II

\section{Introduction}

According to the definition of personality disorders (PDs), they are characterized by enduring maladaptive patterns of behavior, cognition, and inner experience, all exhibited across many contexts and deviating markedly from those accepted by the affected individual's culture. These patterns develop early, are inflexible, and are associated with significant distress or disability. ${ }^{1}$ There are a number of factors that contribute to the development of PDs, ranging from neurobiological and psychosocial, to ethical and cultural factors. ${ }^{2}$ Clinicians are particularly interested in PDs' association with and influence on clinical disorders such as depression.

Depression is a common psychiatric disorder occurring across the life-span, and can be found as comorbid illness in various conditions. ${ }^{3-7}$ Depression and PDs have long been studied, and it is widely accepted that PD has an impact on depression treatment outcomes. ${ }^{8,9}$ However, the distribution of PDs among depressive disorders varies depending on the type of depression, the personality pathology diagnostic system involved, or even the setting of a study. In terms of PDs and types of depressive disorder, Sanderson et al reported a prevalence of PDs among those with major depressive
Correspondence: Nahathai Wongpakaran Department of Psychiatry, Faculty of Medicine, Chiang Mai University, I 10 Intawaroros Road, Tambon Sriphum, Amphoe Muang, Chiang Mai, 50200, Thailand

Tel +66 53945422

Fax +66 53945426

Email nahathai.wongpakaran@cmu.ac.th submit your manuscript | www.dovepress.com

Dovepress

http://dx.doi.org// 0.2/47/NDT.S82884
Neuropsychiatric Disease and Treatment 2015:II 1091-1096

1091

(c) (i) (9) 2015 Wongpakaran et al. This work is published by Dove Medical Press Limited, and licensed under Creative Commons Attribution - Non Commercial (unported, v3.0) BY LC License. The full terms of the License are available at http://creativecommons.org/licenses//by-nd/3.0/. Non-commercial uses of the work are permitted without any further permission from Dove Medical Press Limited, provided the work is properly attributed. Permissions beyond the scope of the License are administered by Dove Medical Press Limited. Intormation on
how to request permission may be found at: http://www.dovepress.com/permissions.php 
disorder (MDD), dysthymic disorder, and double depression, at 50\%, 52\%, and 69\% respectively. The most common types of PD found in their study were avoidant and dependent PDs. ${ }^{10}$ Zimmerman et al meanwhile, found that among patients with MDD, $46 \%$ had at least one PD. Cluster C was prevalent at $21.8 \%$, within which avoidant PD was the most common (14.7\%). ${ }^{11}$ The most common individual PDs associated with MDD were histrionic PD (67\%), avoidant PD (58\%), and obsessive-compulsive PD (49\%).

Corruble et al reviewed the results of studies into PDs up to 1996 and found that cluster C PDs were the most prevalent, while cluster A PDs were the least. The cluster C PDs were the most common and also had the largest variance across studies (ranging from 5\% to 65\%), except for the obsessivecompulsive PD, which had low estimates. ${ }^{12}$ Within cluster B, borderline and histrionic PDs varied the most, within cluster A it was schizoid and schizotypal PDs, while paranoid PD consistently had the lowest scores.

In a recent review of 122 studies carried out by Friborg et al over the period 1988 to 2010, cluster C PDs were the most frequently occurring in unipolar depression; a result which seems to be quite firmly established among the relevant studies. The variation in comorbid proportions showed a similar increasing pattern, varying by $18 \%$ to $35 \%$ for cluster A PDs, by $49 \%$ to $65 \%$ for cluster B PDs, and by between $68 \%$ and $78 \%$ for cluster C PDs. ${ }^{13}$ Among all the PDs, the proportions of schizoid, schizotypal, narcissistic, and antisocial PDs were low $(2 \%-6 \%)$ across all mood disorders. The proportion of histrionic and paranoid PDs was slightly higher, at $6 \%$ to $7 \%$ in MDD and dysthymia. Avoidant PD was more frequently seen in dysthymia, and less often in MDD.

Studies by Corruble et al and Friborg et al found that paranoid PDs varied more than schizoid or schizotypal PDs within cluster A, and obsessive-compulsive PD had equally high variance estimates as the other cluster C PDs. ${ }^{12,13}$ There was a higher variability among borderline, histrionic or narcissistic PDs in cluster B. The variance estimates in dysthymia were less pronounced when compared with MDD. Cluster C PDs, and in particular avoidant PDs, were more noticeable in dysthymia than in MDD. The tool used in a study matters a lot; for example, Huang et al conducted a World Mental Health survey across 13 countries $(n=21,162)$, using an international PD examination tool, and found cluster C PDs have a higher risk of developing mood disorders (odds ratio 9.3, 95\% confidence interval 7.1, 12.2) than in the rest of the clusters. Unfortunately, depressive disorder was not examined separately. ${ }^{14}$
In addition, the race-ethnicity factor plays a role either in the neurobiological preposition or during the diagnosing process for PDs. ${ }^{2,15}$ However, there are few studies of Asian populations, as most research into PDs has been conducted in Europe and North America. ${ }^{13,14,16}$

In this study, the authors aimed to explore the distribution of PDs in patients with depressive disorders, and then compare our results to those found by research in other Asian countries based on comparable conditions, that is, in an outpatient setting, using the Diagnostic and Statistical Manual of Mental Disorders, Fourth Edition, Text Revision (DSM-IV-TR) diagnostic system for Axis I depressive disorder (dysthymic and MDDs), and the Structured Clinical Interview for DSM-IV Axis II Personality Disorders (SCID II) as a diagnostic tool.

\section{Material and methods \\ Study overview}

This study included a cross-sectional analysis, and was an extension of the Thai Study of Affective Disorder (THAISAD) project. It included an observational, 12-month follow-up study of depression treatment outcomes, in which all the participants were $\geq 18$ years of age, received a diagnosis for MDD (single or recurrent; moderate or severe) according to the DSM-IV criteria and without Axis I comorbidity. Details of the processes followed are described elsewhere. ${ }^{6}$ The study's protocol was approved by the ethics committee of the Faculty of Medicine, Chiang Mai University.

\section{Participants}

All 140 participants had been recruited at Chiang Mai University hospital for the THAISAD project, and were approached upon completion of that project. In total, 85 agreed to participate in this study. Among the 85 participants, the mean age was 46.3 years (standard deviation 14.4; range 19-81), with $80 \%$ of the respondents being female, and most of the patients having been educated to at least high school level (63.6\%). In terms of marital status, 27 (31.8\%) were single, 35 were cohabitating or married (41.2\%), and 23 (27.1\%) were living alone (widowed/divorced/separated). In terms of employment, most of the patients were employed (76.5\%). With regard to the diagnosis the patients had received, $71(83.6 \%)$ had been diagnosed with MDD, seven (8.2\%) with dysthymic disorder, and seven (8.2\%) with double depression.

All the patients had been receiving antidepressants, among whom most (68\%) were receiving serotonin selective reuptake inhibitors for their treatment combined with counseling or supportive therapy after the medication regime had been reviewed. 


\section{Measurement}

Trained clinicians used the Mini International Neuropsychiatric Inventory to assess for depression, in accordance with the DSM-IV-TR. The patients were then diagnosed with MDD, dysthymic disorder, and/or double depression (dysthymia superimposed with major depressive episodes). ${ }^{17,18}$

The Thai version of the structured clinical interview for DSM-IV Axis II PDs (T-SCID II) instrument was used to measure for PDs, and was shown to have good reliability. ${ }^{19,20}$ The interviews were carried out after the final visit (at 12 months), and these were undertaken by trained clinicians and an investigator, that is, three psychiatrists and a social worker who had practiced in a psychiatric clinic for more than 20 years. Before the study began, independent rating with trial samples was carried out, and agreement between raters compared. Inter-rating was considered sufficient when the level of agreement between raters reached $100 \%$ (Cohen's kappa 1.00). The raters were unaware of the diagnosis each patient had received.

\section{Statistical analyses}

Data were analyzed in two ways; in a descriptive fashion, with continuous variables illustrated - using the mean \pm standard deviations and medians within a range - as numbers and percentages (categorical data). The statistical significance level for all the tests was set at a $P$-value of $<0.05$ and analyses were performed using IBM SPSS 22.0 statistical software (IBM Corporation, Armonk, NY, USA).

\section{Results}

Table 1 shows the distribution of each PD according to the type of depressive disorder found, most of which were MDD. There was found to be no significant difference in
PDs among individual types of depressive disorder. Those with dysthymic disorder and double depression were shown to have a higher proportion of PDs than those with MDD. Most (30.6\%) had at least one PD (Table 2). Among the PD clusters, cluster B was the most prevalent (27.7\%), and was dominated by borderline PD. It should be noted that passive-aggressive and depressive PDs were found in ten participants, but none of these patients had a single PD, so they were included in the mixed cluster.

Table 3 shows that paranoid, schizotypal, and schizoid cluster A PDs, combined with obsessive-compulsive PD, were most commonly found in the mixed cluster, along with passive-aggressive and depressive PDs.

When comparing PDs found in Asian samples with those from US and Europe, the results were similar, except for cluster A, with one consistent finding among the studies being that any PD (or mixed cluster for the present study) was higher than specific PDs (Table 4).

\section{Discussion}

This is one of just a few studies to have taken place using a sample of patients from Asia suffering from a similar condition, and using DSM criteria to diagnose PDs (SCID II) in an outpatient setting, among patients with depressive disorders. Among the other Asian studies, a study in Iran used a randomized controlled trial (to test the efficacy of behavioral therapy), while the rest (in People's Republic of China, Japan, and Thailand) used the consecutive method to recruit patients..$^{21-23}$ In Japan, a study by Sato et al using DSM-III-R criteria was carried out. ${ }^{23}$ Even though most of the criteria were the same as in this study, some were different eg, depressive PD, so the studies cannot be compared like-for-like. Even though the Japanese study used the same tool - SCID-II, and in similar

Table I Frequency of individuals with PDs according to the type of depressive disorder $(\mathrm{N}=85)$

\begin{tabular}{|c|c|c|c|}
\hline \multirow[t]{2}{*}{ PD } & \multicolumn{3}{|l|}{ Type of depression } \\
\hline & $\begin{array}{l}\text { Major depressive disorder } \\
\text { n (\%) }\end{array}$ & $\begin{array}{l}\text { Dysthymic disorder } \\
\text { n (\%) }\end{array}$ & $\begin{array}{l}\text { Double depression } \\
\text { n (\%) }\end{array}$ \\
\hline Without PD & $17(20)$ & $2(2.4)$ & $\mathrm{I}(\mathrm{I} .2)$ \\
\hline Avoidant & $2(2.4)$ & 0 & 0 \\
\hline Dependent & $\mathrm{I}(\mathrm{I} .2)$ & 0 & 0 \\
\hline Obsessive-compulsive & $6(7.1)$ & $\mathrm{I}(\mathrm{I} .2)$ & $2(2.4)$ \\
\hline Paranoid & $4(4.7)$ & 0 & 0 \\
\hline Schizotypal & $2(2.4)$ & $\mathrm{I}(\mathrm{I} .2)$ & $\mathrm{I}(\mathrm{I} .2)$ \\
\hline Schizoid & $2(2.4)$ & 0 & 0 \\
\hline Histrionic & 0 & 0 & 0 \\
\hline Narcissistic & 0 & 0 & 0 \\
\hline Borderline & $17(20)$ & $2(2.4)$ & $\mathrm{I}(\mathrm{I} .2)$ \\
\hline Antisocial & $I(1.2)$ & 0 & 0 \\
\hline Mixed & $21(24.7)$ & $2(2.4)$ & $2(2.4)$ \\
\hline
\end{tabular}

Abbreviation: PD, personality disorder. 
Table 2 Personality disorder variables $(\mathrm{N}=85)$

\begin{tabular}{|c|c|}
\hline \multirow[t]{2}{*}{ Variable } & \multirow{2}{*}{$\begin{array}{l}\text { Value } \\
\text { Mean } \pm \text { SD } \\
(\min -\max ) \text { OR } n(\%)\end{array}$} \\
\hline & \\
\hline At least one personality disorder & $65(76.5)$ \\
\hline \multicolumn{2}{|l|}{$($ median $=I$, mode $=I)$} \\
\hline 0 & $20(23.5)$ \\
\hline I & $26(30.6)$ \\
\hline 2 & II (12.9) \\
\hline 3 & $13(15.3)$ \\
\hline 4 & $6(7.1)$ \\
\hline 5 & $4(4.7)$ \\
\hline 6 & $3(3.5)$ \\
\hline 7 & $2(2.4)$ \\
\hline \multicolumn{2}{|c|}{ Distribution of personality disorders } \\
\hline \multicolumn{2}{|l|}{ Cluster $A(n=10)$} \\
\hline Paranoid & $4(4.7)$ \\
\hline Schizotypal & $4(4.7)$ \\
\hline Schizoid & $2(2.4)$ \\
\hline \multicolumn{2}{|l|}{ Cluster B $(n=18)$} \\
\hline Histrionic & $0(0)$ \\
\hline Narcissistic & $0(0)$ \\
\hline Borderline & $17(20.0)$ \\
\hline Antisocial & $\mathrm{I}(\mathrm{I} .2)$ \\
\hline \multicolumn{2}{|l|}{ Cluster C ( $n=12)$} \\
\hline Avoidant & $2(2.4)$ \\
\hline Dependent & $\mathrm{I}(\mathrm{I} .2)$ \\
\hline Obsessive-compulsive & $9(10.6)$ \\
\hline \multicolumn{2}{|l|}{ Not otherwise specified } \\
\hline Passive-aggressive & $0(0)$ \\
\hline Depressive & $0(0)$ \\
\hline \multicolumn{2}{|l|}{ Mixed cluster $(n=25)$} \\
\hline Cluster $\mathrm{A}+$ cluster $\mathrm{B}$ & $\mathrm{I}(\mathrm{I} .2)$ \\
\hline Cluster $\mathrm{A}+$ cluster $\mathrm{C}$ & $16(18.8)^{\mathrm{a}}$ \\
\hline Cluster B + cluster C & $2(2.4)^{b}$ \\
\hline Cluster $\mathrm{A}+$ cluster $\mathrm{B}+$ cluster $\mathrm{C}$ & $6(7.1)^{c}$ \\
\hline
\end{tabular}

Notes: ancludes four passive-aggressive PDs and four depressive PDs; bincludes one passive-aggressive PD and one depressive PD; cincludes two passive-aggressive PDs and four depressive PDs.

Abbreviations: PD, personality disorder; SD, standard deviation; OR, odds ratio; min, minimum; max, maximum.

Table 3 PDs in the mixed cluster $(n=25)$

\begin{tabular}{ll}
\hline PD & Value $\mathbf{n}(\%)$ \\
\hline Avoidant & $10(40.0)$ \\
Dependent & $0(0)$ \\
Obsessive-compulsive & $21(84.0)$ \\
Paranoid & $19(76.0)$ \\
Schizotypal & $23(92.0)$ \\
Schizoid & $13(52.0)$ \\
Histrionic & $2(8.0)$ \\
Narcissistic & $4(16.0)$ \\
Borderline & $0(0)^{*}$ \\
Antisocial & $3(12.0)$ \\
Passive-aggressive & $7(28.0)$ \\
Depressive & $9(36.0)$ \\
\hline
\end{tabular}

Note: *Borderline PD was excluded from the list, as it was by nature mixed; no patient had borderline PD alone.

Abbreviation: PD, personality disorder. conditions, the prevalence found varied from this study, which may not be a surprise. As a result, this study will certainly add to the existing knowledge regarding PDs across cultures.

The results in the present study did not show a high prevalence of avoidant PD when compared to other Asian studies, nor those conducted in Europe and the US, while borderline PD has been found to be generally high across cultures, plus as expected is more closely related to depressive symptoms, both biologically and clinically.

The authors also found a high prevalence of mixed cluster PDs, and especially in cluster A and cluster C. This kind of mixed cluster result has not been much reported in previous studies. A mixed cluster, in this sense, is equivalent to what has been described by Tyrer as a "complex (diffuse) personality disorder", which meets the criteria for one or more PDs found within more than one cluster. ${ }^{24} \mathrm{~A}$ mixed cluster may, in fact, reflect PDs in the real world, because it is more prevalent than a single PD, particular among those with depressive disorders. It may also represent a more severe PD and be more complex in terms of the neurobiological vulnerability displayed, a symptom which underscores the presence of this Axis I disorder. Other studies have used the terms "any PD" to account for the existence of such a PD, "PD not otherwise specified", or "PD not included in cluster A, B or C" (eg, passive-aggressive and depressive PDs). ${ }^{14,25} \mathrm{Up}$ until now, there has been no study carried out which focuses on the role a mixed cluster plays in relation to depressive disorder or any other Axis I disorder.

Why was a low prevalence of dependent PD found in this study? One reason might be cultural factors, as proposed by Chen et al who said that people in collectivistic Asian cultures might view dependence as part of normal, appropriate behavior, more so than those living in more individualistic (Western) cultures. ${ }^{26}$ However, although dependent PD has been shown to have a low prevalence in People's Republic of China and Thailand, this has not been the case in Japan.

From this study it is difficult to draw a conclusion with regard to the relationship between race-ethnicity, culture, and PDs, as it is very difficult to control for these other factors. However, what investigators may agree on is that PD is an important factor influencing the prevalence of Axis I disorders. Further investigation is required; therefore, to see how PD influences Axis I disorder symptoms. Finally, further work should be carried out to ascertain the role a combination of any particular PD and Axis I disorders plays in terms of treatment responses, regardless of whether antidepressants or psychotherapy are used. 
Table 4 Comparison of the percentage of PDs found in depressive disorders among global samples

\begin{tabular}{|c|c|c|c|c|c|}
\hline Items & US and Europe ${ }^{13}$ & People's Republic of China ${ }^{22}$ & $\operatorname{Iran}^{21}$ & $J^{J a p a n}{ }^{23}$ & Present study \\
\hline Sample size & 24,867 & 742 & 100 & 118 & 85 \\
\hline Avoidant & 14 & II.I & 6 & 39 & 2.4 \\
\hline Dependent & 9 & 3.5 & 6 & 20 & 1.2 \\
\hline Obsessive-compulsive & 9 & 9.8 & 4 & 23 & 10.6 \\
\hline Paranoid & 6 & 7.1 & 2 & 14 & 4.7 \\
\hline Schizotypal & 5 & 3.2 & 0 & 3 & 4.7 \\
\hline Schizoid & 2 & 2.3 & 0 & 9 & 2.4 \\
\hline Histrionic & 5 & 2.4 & 0 & 14 & 0 \\
\hline Narcissistic & 3 & 2.8 & 0 & 21 & 0 \\
\hline Borderline & 13 & II.I & 2 & 9 & 20.0 \\
\hline Antisocial & - & 0.1 & 0 & 2 & 1.2 \\
\hline Passive-aggressive & - & 3.6 & - & 9 & - \\
\hline Depressive & - & 8.5 & - & - & - \\
\hline Any PD & 44 & 42.2 & - & 59 & 29.4 \\
\hline
\end{tabular}

Abbreviation: PD, personality disorder.

\section{Limitations}

This study had some limitation that should be mentioned. Since the PD diagnosis interviews were carried out at the end of the THAISAD study, this might have impacted upon the reliability of the participants who were still depressed at the time of the interviews. In addition, the sample size was quite small and was chosen using a non-random methodology, and so may not have represented the profile of patients with depressive disorders to be found among the general population. Although the authors initially would like to assess the prevalence of PDs among patients suffering from not only MDD, but also from dysthymia and double depression, the small sample size of the two latter groups, unfortunately, does not allow the study to reach conclusions. Hence, the study is limited to the assessment of PDs in MDD, and requires further study among non-MDD patients.

\section{Acknowledgments}

This study was part of the THAISAD study conducted at Chiang Mai University research site, and was funded by the National Research Council of Thailand, and was coordinated and supported by the Medical Research Network of the Consortium of Thai Medical School. Additional funding for the research was provided by the Faculty of Medicine, Chiang Mai University.

\section{Author contributions}

All authors have contributed to conception and participated on the protocol designed, and acquisition of data. TW performed the analysis of the available data. NW drafted the manuscript and the rest authors revised it critically for important intellectual content. All authors read and approved the final manuscript and agreed to be accountable for all aspects of the work in ensuring that questions related to the accuracy or integrity of any part of the work are appropriately investigated and resolved.

\section{Disclosure}

The authors have no conflicts of interest to disclose.

\section{References}

1. American Psychiatric Association. Diagnostic and Statistical Manual of Mental Disorders, Fouth Edition, Text Revision. Washington, DC: American Psychiatric Association; 2000.

2. Huang B, Grant BF, Dawson DA, et al. Race-ethnicity and the prevalence and co-occurrence of Diagnostic and Statistical Manual of Mental Disorders, Fourth Edition, alcohol and drug use disorders and Axis I and II disorders: United States, 2001 to 2002. Compr Psychiatry. 2006;47(4): 252-257.

3. Carotenuto M, Esposito M, Parisi L, et al. Depressive symptoms and childhood sleep apnea syndrome. Neuropsychiatr Dis Treat. 2012;8: 369-373.

4. Esposito M, Roccella M, Gallai B, et al. Maternal personality profile of children affected by migraine. Neuropsychiatr Dis Treat. 2013;9:1351-1358.

5. Esposito M, Gallai B, Roccella M, et al. Anxiety and depression levels in prepubertal obese children: a case-control study. Neuropsychiatr Dis Treat. 2014;10:1897-1902.

6. Wongpakaran T, Wongpakaran N, Pinyopornpanish M, et al. Baseline characteristics of depressive disorders in Thai outpatients: findings from the Thai Study of Affective Disorders. Neuropsychiatr Dis Treat. 2014;10: 217-223.

7. Wongpakaran N, Wongpakaran T. Prevalence of major depressive disorders and suicide in long-term care facilities: a report from northern Thailand. Psychogeriatrics. 2012;12(1):11-17.

8. Newton-Howes G, Tyrer P, Johnson T. Personality disorder and the outcome of depression: meta-analysis of published studies. $\mathrm{Br}$ $J$ Psychiatry. 2006;188:13-20.

9. Newton-Howes G, Tyrer P, Johnson T, et al. Influence of personality on the outcome of treatment in depression: systematic review and meta-analysis. J Pers Disord. 2014;28(4):577-593.

10. Sanderson WC, Wetzler S, Beck AT, Betz F. Prevalence of personality disorders in patients with major depression and dysthymia. Psychiatry Res. 1992;42(1):93-99. 
11. Zimmerman M, Rothschild L, Chelminski I. The prevalence of DSM-IV personality disorders in psychiatric outpatients. Am J Psychiatry. 2005; 162(10):1911-1918.

12. Corruble E, Ginestet D, Guelfi JD. Comorbidity of personality disorders and unipolar major depression: a review. J Affect Disord. 1996; 37(2-3):157-170.

13. Friborg O, Martinsen EW, Martinussen M, Kaiser S, Overgård KT, Rosenvinge JH. Comorbidity of personality disorders in mood disorders: a meta-analytic review of 122 studies from 1988 to 2010. J Affect Disord. 2014;152-154:1-11.

14. Huang Y, Kotov R, de Girolamo G, et al. DSM-IV personality disorders in the WHO World Mental Health Surveys. Br J Psychiatry. 2009; 195(1):46-53.

15. Shariat SV, Assadi SM, Noroozian M, et al. Psychopathy in Iran: a cross-cultural study. J Pers Disord. 2010;24(5):676-691.

16. Ryder AG, Sun J, Dere J, Fung K. Personality disorders in Asians: summary, and a call for cultural research. Asian J Psychiatr. 2014;7(1): 86-88.

17. Sheehan D, Lecrubier Y, Sheehan KH, et al. The Mini-International Neuropsychiatric Interview (M.I.N.I.): the development and validation of a structured diagnostic psychiatric interview for DSM-IV and ICD-10. J Clin Psychiatry. 1998;59 Suppl 20:22-23.

18. Kittirattanapaiboon P, Khamwongpin M. [The Validity of the Mini International Neuropsychiatric Interview (M.I.N.I.)-ThaiVersion]. J Ment Health Thai. 2005;13(3):125-135. Thai.
19. First MB, Gibbon M, Spitzer RL, Williams JBW, Benjamin LS. Structured Clinical Interview for DSM-IV Axis II Personality Disorder (SCID-II), User's Guide. Washington, DC: American Psychiatric Press; 1997.

20. Wongpakaran T, Wongpakaran N. Structured Clinical Interview for DSM-IV Axis II Personality Disorders (SCID-II) Thai version. Bangkok: IGroup Press Co. Ltd; 2012.

21. Moradveisi L, Huibers MJ, Renner F, Arasteh M, Arntz A. The influence of comorbid personality disorder on the effects of behavioural activation vs. antidepressant medication for major depressive disorder: results from a randomized trial in Iran. Behav Res Ther. 2013;51(8):499-506.

22. Zhang T, Wang L, Good MJ, et al. Prevalence of personality disorders using two diagnostic systems in psychiatric outpatients in Shanghai, China: a comparison of uni-axial and multi-axial formulation. Soc Psychiatry Psychiatr Epidemiol. 2012;47(9):1409-1417.

23. Sato T, Sakado K, Uehara T, Sato S, Nishioka K, Kasahara Y. Personality disorder diagnoses using DSM-III-R in a Japanese clinical sample with major depression. Acta Psychiatr Scand. 1997;95(5):451-453.

24. Tyrer P. Personality Disorders: Diagnosis, Management and Course. 2nd ed. London: Arnold Publishers Ltd.; 2000.

25. Lenzenweger MF. Epidemiology of personality disorders. Psychiatr Clin North Am. 2008;31(3):395-403.

26. Chen Y, Nettles ME, Chen SW. Rethinking dependent personality disorder: comparing different human relatedness in cultural contexts. J Nerv Ment Dis. 2009;197(11):793-800.
Neuropsychiatric Disease and Treatment

\section{Publish your work in this journal}

Neuropsychiatric Disease and Treatment is an international, peerreviewed journal of clinical therapeutics and pharmacology focusing on concise rapid reporting of clinical or pre-clinical studies on a range of neuropsychiatric and neurological disorders. This journal is indexed on PubMed Central, the 'PsycINFO' database and CAS,

\section{Dovepress}

and is the official journal of The International Neuropsychiatric Association (INA). The manuscript management system is completely online and includes a very quick and fair peer-review system, which is all easy to use. Visit http://www.dovepress.com/testimonials.php to read real quotes from published authors. 